\title{
On Conditional Expectations Arising from Group Actions
}

\author{
M. Frank, V. M. Manuilov and E. V. Troitsky
}

\begin{abstract}
Induced conditional expectations of finite index on crossed product $\mathrm{C}^{*}$-algebras are considered which are non-algebraically of finite index. The characteristics of actions of (amenable) topological groups on compact Hausdorff spaces $X$ are investigated, ensuring the appearance of a well-defined induced conditional expectation on the corresponding commutative $\mathrm{C}^{*}$-algebra $\mathrm{C}(X)$ and its property to be of finite index. For this purpose Hilbert $\mathrm{C}^{*}$-module and topological techniques are used. Special emphasis is placed on discrete group actions.
\end{abstract}

Keywords: Group actions on compact spaces, induced conditional expectations of finite index, crossed product $C^{*}$-algebras, commutative $C^{*}$-algebras

AMS subject classiflcation: Primary 46 L 99, secondary 46 H 25

In the light of recent results by $E$. Kirchberg and the first author [6] we generalize some interesting results of Mahmood Khoshkam [10] concerning special conditional expectations on crossed product $\mathrm{C}^{*}$-algebras which are of finite index in the sense of $Y$. Watatani's algebraic definition [20] to the broader setting of similar conditional expectations $E$ on them which are of finite index in the sense of M. Baillet, Y. Denizeau and J.-F. Havet [3], i.e. those for which there exists a finite real number $K \geq 1$ so that the mapping $(K \cdot E-\mathrm{id})$ is positive.

Subsequently we consider actions of amenable infinite discrete or topological groups on locally compact Hausdorff spaces which give rise to conditional expectations of finite index. In these cases the action of an amenable topological group $G$ can always be replaced by a suitable action of the completely disconnected group arising as the factor group of $G$ by its connected component of the identity. The influence of the maximal length of orbit in $X$ under the group action on the characteristic constant $K\left(E_{G}\right)$ of the derived conditional expectation of finite index on $\mathrm{C}_{0}(X)$ is described.

In addition we analyze the constructions of conditional expectations of finite index on commutative $\mathrm{C}^{*}$-algebras $\mathrm{C}(X)$ given by $\mathrm{Y}$. Watatani [3: Propositions 2.8 .1 and 2.8.2], which arise from actions of finite groups on compact Hausdorff spaces $X$. Special attention is paid to the close interrelation between non-maximal orbits in $X$ under the group action and of the non-algebraic character of the corresponding conditional expectation of finite index. For these investigations we use group action and Hilbert

M. Frank: University of Leipzig, Inst. Math., D - 04109 Leipzig

V. M. Manuilov: Moscow State University, Dept. Mech. Math., 119899 Moscow, Russia

E. V. Troitsky: Moscow State University, Dept. Mech. Math., 119899 Moscow, Russia

ISSN 0232-2064 / $\$ 2.50$ (C) Heldermann Verlag Berlin 
$\mathrm{C}^{*}$-module techniques. Examples are used to illustrate the phenomena appearing for discrete group actions.

We make use of some basic notions and results from the theory of Hilbert $C^{*}$ modules during our considerations to streamline the explanations. For an introduction to this theory we refer the reader to the papers by W. L. Paschke [12] and M. A. Rieffel $[17]$.

\section{On liftings of conditional expectations of finite index to crossed products}

Throughout the present section we only deal with discrete groups $G$. If a group $G$ acts on a $C^{*}$-algebra $A$ as a group of *-automorphisms, we denote the full and the reduced crossed product $C^{*}$-algebras by $A \rtimes G$ and $A \rtimes_{r} G$ respectively (cf. $([4,14,19]$ ). We consider conditional expectations $E: A \rightarrow B \subseteq A$ on $\mathrm{C}^{*}$-algebras $A$, i.e. projections of norm one with range $C^{*}$-subalgebra $B \subseteq A$ (see $[14,19]$ for detailed results). The $C^{*}$ algebra $A$ can always be assumed to be unital without loss of generality since $E$ can be continued to a normal conditional expectation $E^{* *}$ on the bidual $\mathrm{W}^{*}$-algebra $A^{* *}$ of $A$ with range $\mathrm{C}^{*}$-algebra $E^{* *}\left(A^{* *}\right) \subseteq A^{* *}$ which is *-isomorphic to the bidual $\mathrm{W}^{*}$-algebra $B^{* *}$ of $B$, whereby the reduction of $E^{* *}$ to the canonical embedding $A \hookrightarrow A^{* *}$ recovers $E$. A conditional expectation $E$ is said to be faithful if $E\left(x^{*} x\right)=0$ implies $x=0$ for $x \in A$. In this case both $A$ and $E(A)=B$ share a common identity. To consider both conditional expectations $E: A \rightarrow B \subseteq A$ and actions $\alpha$ of groups $G$ on the $C^{*}$-algebras involved, we suppose that the group action $\alpha$ of $G$ by *-automorphisms on $A$ commutes with the conditional expectation $E$.

We shall investigate conditional expectations of finite index both in the sense of Y. Watatani [20] and in the sense of M. Baillet, Y. Denizeau and J.-F. Havet [3]. If we consider $W^{*}$-factors and normal conditional expectations on them with factor image $W^{*}$ algebra, both notions reduce to the classical index notion of V. Jones, M. Pimsner and S. Popa $[3,9,15]$. A conditional expectation $E: A \rightarrow B \subseteq A$ is said to be algebraically of finite index if there exist elements $\left\{u_{1}, \ldots, u_{n}\right\} \subset A$, a quasi-basis, such that the equality $x=\sum_{i=1}^{n} u_{i} E\left(u_{i}^{*} x\right)$ is valid for every $x \in A$. In this case the index is defined by $\operatorname{Ind}(E)=\sum_{i=1}^{n} u_{i} u_{i}^{*}$, which is a positive invertible element in the center of $A$ such that Ind $(E) \geq 1_{A}$, and it does not depend on the choice of the quasi-basis $\left\{u_{1}, \ldots, u_{n}\right\}$ inside $A$ and in particular not on the number of its elements [20]. From another point of view the existence of a finite quasi-basis for some conditional expectation $E: A \rightarrow B \subseteq A$ is equivalent to the algebraic statement that $A$ is a finitely generated projective (or, equivalently, Hilbert) $B$-module.

There is another interesting class of conditional expectations $E: A \rightarrow B \subseteq A$ - those for which there exists a finite real number $K \geq 1$ such that the mapping $\left(K \cdot E-\mathrm{id}_{A}\right)$ is a positive map on $A$. They have the following remarkable property:

Proposition 1.1. Let $A$ be a $C^{*}$-algebra and $E: A \rightarrow B \subseteq A$ be a conditional expectation with fixed point set $B$. There then exists a finite real number $K \geq 1$ such that the mapping $\left(K \cdot E-\mathrm{id}_{A}\right)$ is positive if and only if $E$ is faithful and the (right) pre-Hilbert $B$-module $\left\{A, E\left(\langle\cdot ;\rangle_{A}\right)\right\}$ is complete with respect to the norm $\left\|E\left(\langle\cdot, \cdot\rangle_{A}\right)\right\|_{B}^{\frac{1}{2}}$ (where $\langle a, b\rangle_{A}=a^{*} b$ for $\left.a, b \in A\right)$. 
Proof. If $A$ is complete with respect to the norm $\left\|E\left(\langle\cdot, \cdot\rangle_{A}\right)\right\|_{B}^{\frac{1}{2}}$, then by the general theory of Banach spaces there exists a number $K$ such that $K\left\|E\left(x^{*} x\right)\right\| \geq\left\|x^{*} x\right\|$ holds for every $x \in A$. Set $x=a\left(\varepsilon+E\left(a^{*} a\right)\right)^{-\frac{1}{2}}$ for $a \in A$ and $\varepsilon>0$, and observe that

$$
\left(\varepsilon+E\left(a^{*} a\right)\right)^{-\frac{1}{2}} \cdot E\left(a^{*} a\right) \cdot\left(\varepsilon+E\left(a^{*} a\right)\right)^{-\frac{1}{2}} \leq 1_{A} .
$$

The inequality

$$
K \cdot 1_{A} \geq\left(\varepsilon+E\left(a^{*} a\right)\right)^{-\frac{1}{2}} \cdot a^{*} a \cdot\left(\varepsilon+E\left(a^{*} a\right)\right)^{-\frac{1}{2}}
$$

holds. Multiplying by $\left(\varepsilon+E\left(a^{*} a\right)\right)^{\frac{1}{2}}$ from both sides we arrive at $K \cdot\left(\varepsilon+E\left(a^{*} a\right)\right) \geq a^{*} a$ for every $a \in A$ and $\varepsilon>0$. This yields the other statement. The converse is obvious by spectral theory and by the inequality $\|E(x)\| \leq\|x\|$ which is valid for every $x \in A$

The difference between the two types of conditional expectations of being of finite index arises in the way the Hilbert $B$-module $\left\{A, E\left(\langle\cdot, \cdot\rangle_{A}\right)\right\}$ is generated, finitely or infinitely. (see [6], [20: Propositions 2.8.1 and 2.8.2], and Section 3). The results of collaboration between $E$. Kirchberg and the first author [6] showed that conditional expectations $E: A \rightarrow B \subseteq A$ of finite index have been identified with those satisfying the following equivalent conditions: for a conditional expectation $E$ on a (unital) $\mathrm{C}^{*}$. algebra $A$ there exists a real number $K \geq 1$ such that the mapping $\left(K \cdot E-\mathrm{id}_{A}\right)$ is positive if and only if there exists a real number $L \geq 1$ such that the mapping $\left(L \cdot E-\mathrm{id}_{A}\right)$ is completely positive, if and only if $E$ is faithful and $A$ is complete with respect to the norm $\left\|E\left((\cdot, \cdot\rangle_{A}\right)\right\|^{\frac{1}{2}}$ (where $\langle a, a\rangle_{A}=a^{*} a$ for $a \in A$ ). Setting

$$
K(E)=\inf \left\{K:\left(K \cdot E-\mathrm{id}_{A}\right) \text { is positive on } A\right\}
$$

and

$$
L(E)=\inf \left\{L:\left(L \cdot E-\mathrm{id}_{A}\right) \text { is completely positive on } A\right\}
$$

the estimate $K(E) \leq L(E) \leq K(E) \cdot[K(E)]$ can be shown, where [.] denotes the entire part of a real number. Moreover, in addition to the properties of this class of conditional expectations obtained in [1], normal conditional expectations of finite index on $W^{*}$-algebras commute with the (abstract) projections of $W^{*}$-algebras to their finite, infinite, discrete and continuous type $\mathrm{I}$, type $\mathrm{II}_{1}$, type $\mathrm{II}_{\infty}$ and type III parts, i.e. they respect and preserve these $W^{*}$-decompositions in full. Additionally, the index value and the Jones' tower always exist in this case. In the general $\mathrm{C}^{*}$-case the index value of $E$ can only be obtained as an element of the center of the bidual $\mathrm{W}^{*}$-algebra $A^{* *}$ of $A$ extending $E$ to its canonical normal conditional expectation $E^{* *}: A^{* *} \rightarrow B^{* *} \subseteq A^{* *}$ (see $[4,19]$ and Section 3 ). However, if the index value belongs to the image $\mathrm{C}^{*}$-algebra $B$, the Jones' tower can be shown to exist.

These observations of [6] extend and augment the results of M. Pimsner, S. Popa [15], M. Baillet, Y. Denizeau and J.-F. Havet [3], Y. Watatani [20], P. Jolissaint [8], E. Andruchow and D. Stojanoff $[1,2]$ and others, closing the gap of knowledge separating Proposition 3.3 and Theorem 3.5 of [3]. There are examples of conditional expectations of finite index which are not of the purely algebraic type investigated by $Y$. Watatani (cf. [20: Proposition 2.8.2] and Section 3). A large class of these examples is closely 
related to group actions on compact Hausdorff spaces and the related $\mathrm{C}^{*}$-algebras, as well as to crossed products.

In the special situation of a conditional expectation $E: A \rightarrow B \subseteq A$ and a discrete group $G$ acting on $A$, where the action $\alpha$ of $G$ and the mapping $E$ are supposed to commute, M. Khoshkam showed that there exist canonically induced conditional expectations $\tilde{E}$ from $A \rtimes G$ to $B \rtimes G \subseteq A \rtimes G$, respectively $\tilde{E}_{r}$ from $A \rtimes_{r} G$ to $B \rtimes_{r} G \subseteq A \rtimes_{r} G$ [10: Remark 2.6]. They arise from the projection of $l^{1}(G, A)$ onto $l^{1}(G, B)$ defined by $f \rightarrow E \circ f$.

Now we extend the result of $M$. Khoshkam [10: Theorem 2.8] that the additional property of one of the three interrelated conditional expectations $E, \tilde{E}_{r}$ and $\tilde{E}$ of being algebraically of finite index implies the same property for the other two mappings. We would like to consider the more general case of conditional expectations of finite index (cf. [3]) possessing a finite real number $K \geq 1$ such that the mapping $(K \cdot E-$ id) is positive.

Proposition 1.2. Let $E: A \rightarrow B \subseteq A$ be a conditional expectation of finite index with characteristic number $K(E)$. Then $E$ is of finite inde $\dot{x}$ if and only if $\tilde{E}_{r}$ is of finite index, if and only if $\tilde{E}$ is of finite index. The equality $K\left(\tilde{E}_{\mathrm{r}}\right)=K(\tilde{E})=K(E)$ holds for their characteristic numbers. In particular, $\tilde{E}$ is faithful in this case.

If, moreover, either $E, \tilde{E}_{\mathrm{r}}$ or $\tilde{E}$ is algebraically of finite index in the sense of $Y$. Watatani [20], then both the other conditional expectations possess the same property.

Proof. If $E$ is faithful, then $\tilde{E}_{r}$ is faithful too (and vice versa), since the conditional expectations $E_{0}: A \rtimes_{r} G \rightarrow A$ and $E_{0}^{\prime}: B \rtimes_{r} G \rightarrow B$ given by the evaluation at the identity of $G$ are faithful and the equality $E_{0}^{\prime} \circ \tilde{E}_{r}=E \circ E_{0}$ holds. Because of the normdensity of $A$ in the pre-Hilbert $B$-module $\{A, E(\langle c d o t, \cdot\rangle)\}$ the action of $G$ extends to its norm-completion $\mathcal{E}_{E}$ for every faithful conditional expectation ' $E$. Similarly, $\mathcal{E}_{E} \rtimes_{r} G$ can be formed. By [10: Propposition 2.7] both the Hilbert $B \rtimes_{r} G$-modules $\mathcal{E}_{\bar{E}_{\mathrm{r}}}$ and $\mathcal{E}_{E} \rtimes_{r} G$ are isometrically isomorphic.

Furthermore, suppose $E$ is of finite index in the sense of [3]. By Proposition 1.1 the Hilbert $B$-module $\mathcal{E}_{E}$ coincides with $A$. Consequently, the Hilbert $B \rtimes_{r} G$-module $\mathcal{E}_{\tilde{E}_{r}}$ coincides with $A \rtimes_{r} G$, and Proposition 1.1 shows again the existence of a finite real number $K\left(\tilde{E}_{r}\right) \geq 1$ such that the mapping $\left(K\left(\tilde{E}_{r}\right) \cdot \tilde{E}_{r}-\mathrm{id}_{A \rtimes_{r} G}\right)$ is positive, i.e. $\tilde{E}_{r}$ is of finite index. The conclusions work equally well in the converse direction.

If $\tilde{E}$ is of finite index, it obviously has to be faithful. Hence [20: Proposition 2.7] and the assertions above apply again, and $E$ turns out to be of finite index, too. Conversely, suppose $E$ to be of finite index. This means the validity of the inequality

$$
K(E) \cdot E\left(a^{*} a\right) \geq a^{*} a
$$

for every $a \in A$. Denoting by $\lambda_{g, a} \in l^{1}(G, A)$ the element giving $a \in A$ at $g \in G$ and zero elsewhere, we obtain

$$
\begin{aligned}
\tilde{E}\left(\left(\sum_{i=1}^{n} \lambda_{g_{i}, a_{i}}\right) *\left(\sum_{i=1}^{n} \lambda_{g_{i}, a_{i}}\right)^{*}\right)(e) & =\tilde{E}\left(\sum_{i=1}^{n} \lambda_{g_{i}, a_{i}} * \lambda_{g_{i}, a_{i}}^{*}\right)(e)=\sum_{i=1}^{n} E\left(a_{i} a_{i}^{*}\right) \\
& =E\left(\sum_{i=1}^{n} a_{i} a_{i}^{*}\right) \geq K(E) \cdot \sum_{i=1}^{n} a_{i} a_{i}^{*} \geq 0,
\end{aligned}
$$


which implies the faithfulness of $\tilde{E}$ on $l^{1}(G, A)$ since the inequality above is preserved for all elements of $l^{1}(G, A)$ by continuity. Consequently, $\tilde{E}$ is faithful on $A \rtimes G$. To finish, we apply again Proposition 1.1 and the assertions above to $\tilde{E}$, which proves $\tilde{E}$ to be of finite index.

The remaining part of the proof is concerned with the estimates of the characteristic numbers $K(E)$ for the three conditional expectations $E, \tilde{E}_{r}$ and $\tilde{E}$ which are now known to be of finite index (and hence, faithful) if and only if one of them is. Again, let $E_{0}$, $E_{0}^{\prime}$ be those conditional expectations $E_{0}: A \rtimes_{(r)} G \rightarrow A, E_{0}^{\prime}: B \rtimes_{(r)} G \rightarrow B$ which arise as evaluation at the identity of $G$. Since both $\tilde{E}$ and $\tilde{E}_{r}$ are already shown to be faithful in the case that $E$ is of finite index the equality

$$
E_{0}^{\prime} \circ \tilde{E}_{(r)} \equiv E \circ E_{0}
$$

is valid. Note that $E_{0}$ and $E_{0}^{\prime}$ are faithful by [5], and that the restriction of $E_{0}$ to $B \rtimes_{(r)} G$ yields $E_{0}^{\prime}$. By (1) we obtain the identity

$$
E_{0}^{\prime} \circ\left(K(E) \cdot \tilde{E}_{(r)}-\mathrm{id}_{A \rtimes_{(r)} G}\right) \equiv\left(K(E) \cdot E-\mathrm{id}_{A}\right) \circ E_{0}
$$

valid on $A \rtimes_{(r)} G$. Suppose $E$ is of finite index with characteristic number $K(E)>1$. $\left(K(E)=1\right.$ implies $E=\mathrm{id}_{A}$.) By assumption

$$
\left(\left(K(E) \cdot E-\mathrm{id}_{A}\right) \circ E_{0}\right)(a)=\left(E_{0}^{\prime} \circ\left(K(E) \cdot \tilde{E}_{(r)}-\mathrm{id}_{\left.A \diamond_{(r)} G\right)}\right)(a) \geq 0\right.
$$

for every $a \in\left(A \rtimes_{(r)} G\right)_{h}^{+}$. Since $E_{0}^{\prime}$ is faithful and has a special mapping rule, the

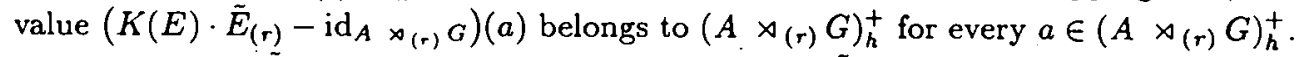
This implies $K\left(\tilde{E}_{(r)}\right) \leq K(E)$. Conversely, suppose $\tilde{E}_{(r)}$ to be of finite index with characteristic number $K\left(\tilde{E}_{(r)}\right)>1$. Then

$$
\left(E_{0}^{\prime} \circ\left(K(E) \cdot \tilde{E}_{(r)}-\mathrm{id}_{A \times(r)} G\right) b i g\right)(a)=\left(\left(K(E) \cdot E-\mathrm{id}_{A}\right) \circ E_{0}\right)(a) \geq 0
$$

for every $a \in\left(A \rtimes_{(r)} G\right)_{h}^{+}$. Since $E_{0}$ is a faithful positive mapping, we derive the

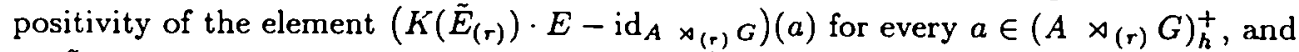
$K\left(\tilde{E}_{(r)}\right) \geq K(E)$. This proves the equality $K\left(\tilde{E}_{r}\right)=K(\tilde{E})=K(E)$

The following assertion extends another result of $M$. Khoshkam about conditional expectations which are algebraically of finite index to the case of those of finite index (cf. [10: Proposition 3.5]).

Proposition 1.3. Let $A \supseteq B \supseteq C$ be $C^{*}$-algebras with the same unit. Suppose the maps $E: A \rightarrow B \subseteq A$ and $F: B \rightarrow C \subseteq B$ are faithful conditional expectations relating these three $C^{*}$-algebras. Then $F \circ E: A \rightarrow C \subseteq A$ has finite index if and only if $E$ and $F$ both have finite indices. Moreover, the inequality $K(F \circ E) \leq K(F) \cdot K(E)$ is valid. Finally, the composition $F \circ E$ is algebraically of finite index if and only both $F$ and $E$ are so.

Proof. Suppose $E$ and $F$ to be of finite index in the sense of [6], i.e. there exists real-numbers

$$
K(E)=\inf \left\{K:\left(K \cdot E-\mathrm{id}_{A}\right) \text { is positive on } A\right\} \geq 1
$$


and

$$
K(F)=\inf \left\{K:\left(K \cdot F-\mathrm{id}_{B}\right) \text { is positive on } B\right\} \geq 1 .
$$

Since $K(E)=1$ implies $E=\mathrm{id}_{A}$ we may assume $K(E)>1$ without loss of generality. Because the inequality $K(F) \cdot F \geq \mathrm{id}_{B}$ is valid by assumption, we conclude that

$$
\left(K(F) \cdot K(E) \cdot F \circ E-\mathrm{id}_{A}\right) \geq K(E) \cdot E-\mathrm{id}_{A} \geq 0
$$

which implies $F \circ E$ to be of finite index.

Conversely, let $F \circ E$ be of finite index. Then there exists a finite real number $K(F \circ E)$ such that the map $\left(K(F \circ E) \cdot F \circ E-\mathrm{id}_{A} b i g\right)$ is positive. Applying the positive map $E$ from the left we obtain

$$
0 \leq E \circ\left(K(F \circ E) \cdot F \circ E-\mathrm{id}_{A}\right)=\left(K(F \circ E) \cdot F-\mathrm{id}_{B}\right) \circ E,
$$

which implies the positivity of the map $\left(K(F \circ E) \cdot F-\mathrm{id}_{B}\right)$ for the finite real number $K(F \circ E)$, and hence proves $F$ of being of finite index.

Furthermore, assume that $E$ is not of finite index. Equivalently, there exists a sequence $\left\{a_{i}\right\}_{i \in \mathbb{N}} \subset A_{h}^{+}$such that the sequence $\left\{\left\|E\left(a_{i}\right)\right\|\right\}_{i \in \mathbb{N}}$ is bounded, but the sequence $\left\{\left\|a_{i}\right\|\right\}_{i \in \mathbf{N}}$ is not. Then the sequence $\left\{\left\|F\left(E\left(a_{i}\right)\right)\right\|\right\}_{i \in \mathbf{N}}$ is bounded since $F$ was shown to be of finite index. However, $F\left(E\left(a_{i}\right)\right)=(F \circ E)\left(a_{i}\right)$, and $F \circ E$ was supposed to be of finite index, too. Consequently, the sequence $\left\{\left\|a_{i}\right\|\right\}_{i \in N}$ has to be bounded. in contravention to its choice. The only way out is the assumption of the existence of a finite real number $K(E)$ such that the inequality $K(E) \cdot\|E(a)\| \geq\|a\|$ holds for every $a \in A_{h}^{+}$. This condition is equivalent to the property of $E$ being of finite index.

For the special case of $E$ and $F$ or $F \circ E$ being algebraically of finite index we refer to M. Khoshkam's result. The inequality $K(F \circ E) \leq K(F) \cdot K(E)$ can be derived from (2) above. An example below shows that equality docs in general not hold

Example 1.4. Consider the faithful conditional expectations of finite index

$$
\begin{aligned}
E: \mathrm{M}_{2}(\mathbf{C}) & \rightarrow \mathbf{C}^{2}, \quad E:\left(\begin{array}{ll}
a & b \\
c & d
\end{array}\right) \rightarrow\left(\begin{array}{ll}
a & 0 \\
0 & d
\end{array}\right) \\
F: \mathbf{C}^{2} & \rightarrow \mathbf{C}, \quad F:\left(\begin{array}{ll}
a & 0 \\
0 & d
\end{array}\right) \rightarrow \frac{a+d}{2} \cdot\left(\begin{array}{ll}
1 & 0 \\
0 & 1
\end{array}\right) .
\end{aligned}
$$

The corresponding characteristic numbers are $K(E)=K(F)=K(F \circ E)=2$, and, therefore, $K(F \circ E)<K(F) \cdot K(E)$.

For a discrete group $G$ and a subgroup $H \subseteq G$ we can define conditional expectations $E^{H}$ from $A \rtimes G$ onto $A \rtimes H$, respectively $E_{r}^{H}$ ) from $A \rtimes_{r} G$ onto $A \rtimes_{r} H$, induced by the canonical projection of $l^{1}(G, A)$ onto $l^{1}(H, A)$ (see [10] for explanations). By [10: Theorem 3.4] the conditional expectations $E^{H}$ and $E_{r}^{H}$ have finite index if and only if $[G: H]<\infty$, and $\operatorname{Ind}\left(E_{(r)}^{H}\right)=[G: H]$ in this case. Applying Proposition 1.3 to the conditional expectation $E_{(r)}^{H} \circ \tilde{E}_{(r)}: A \rtimes_{(r)} G \rightarrow B \rtimes_{(r)} H$ we can formulate the following result (cf. [10: Theorem 3.6] for the algebraic case):

Corollary 1.5. In the situation described the conditional expectation $E_{(r)}^{H} \circ \tilde{E}_{(r)}$ is of finite index if and only if $E$ is of finite index and $[G: H]<\infty$. It is algebraically of finite index if and only if $E$ is algebraically of finite index and $[G: H]<\infty$. In the latter case the indices can be counted and $\left.\operatorname{Ind}\left(\tilde{E}_{(r)}\right)\right)=[G: H] \cdot \operatorname{Ind}(E)$. 


\section{Conditional expectations arising from topological group actions}

For a locally compact Hausdorff space $X$ and a continuous action of a topological group $G$ on $X$ we denote the $C^{*}$-subalgebra of $G$-invariant continuous functions on $X$ vanishing at infinity by $\mathrm{C}_{0}^{G}(X)$ and the stabilizer subgroup of $x \in X$ by $G_{x}=\{g \in G: g x=x\}$. We want to consider situations for which the group action of $G$ on $X$ gives rise to a well-defined conditional expectation $E_{G}$ on the corresponding $\mathrm{C}^{*}$-algebra $\mathrm{C}_{0}(X)$. For this purpose we need the following definitions:

Definition 2.1. We say that an action of a topological group $G$ on a locally compact Hausdorff space $X$ is uniformly continuous if for every point $x \in X$ and every neighbourhood $U_{x}$ of $x$ there exists a neighbourhood $V_{x}$ of $x$ such that $g\left(V_{x}\right) \subseteq U_{x}$ for every $g \in G_{x}$.

Example 2.2. Let the space $X \subset \mathbb{R}^{2}$ be defined in polar coordinates $(r, \phi)$ by

$$
X=\left\{\left(\frac{1}{n}, \frac{\pi}{m}\right): n, m \in \mathbb{N}\right\} \cup\left\{\left(\frac{1}{n}, 0\right): n \in \mathbb{N}\right\} \cup\{(0,0)\}
$$

As $X$ is a closed bounded subset in $\mathbb{R}^{2}$ it is a compact normal space. For the sake of convinience we denote its components by

$$
\begin{array}{r}
X_{m}=\left\{\left(\frac{1}{n}, \frac{\pi}{m}\right): n \in \mathbb{N}\right\} \cup\{(0,0)\} \quad(m \in \mathbb{N}) \\
X_{0}=\left\{\left(\frac{1}{n}, 0\right): n \in \mathbb{N}\right\} \cup\{(0,0)\}, \quad X=\bigcup_{m=0}^{\infty} X_{m} .
\end{array}
$$

Let $G \subseteq \oplus_{1}^{\infty} \mathrm{Z}_{2}$ be the group of sequences $g=\left(g_{1}, \ldots, g_{k}, \ldots\right)$ with entries equal to zero or one, where only a finite number of elements differs from zero. By $g_{(k)}$ we denote the sequence with the unique non-zero element on the $k$-th place. Define an action of $G$ on $X$ by the following rules:

(i) If $x \in X_{m}$ and $m \neq k$, then $g_{(k)} x=x$,

(ii) If $x \in X_{k}, x=\left(\frac{1}{n}, \frac{\pi}{k}\right)$ and $n \neq 1, n \neq k$, then again $g_{(k)} x=x$,

(iii) $g_{(k)}$ transposes the points $\left(1, \frac{\pi}{k}\right)$ and $\left(\frac{1}{k}, \frac{\pi}{k}\right)$.

Under the action of $G$, the orbit of every point of $X \backslash X_{0}$ consists of exactly two points, and the points of $X_{0}$ are fixed. Although the described action is continuous (when $G$ is considered as a discrete group), this action does not satisfy the condition of uniform continuity as every neighbourhood of the origin contains points which are moved by some element of $G$ to points located at a distance from the origin equal to one.

Note that a continuous action of a compact group is always uniformly continuous.

Definition 2.3. An action of an amenable topological group $G$ on a locally compact Hausdorff space $X$ generates a conditional expectation $E_{G, a m}$ on $\mathrm{C}_{0}(X)$ if the formula

$$
E_{G, a m}(f)(x)=m_{G}\left(f\left(g^{-1} x\right)\right)
$$


defines a continuous function for any $f \in \mathrm{C}_{0}(X)$ and for some fixed (left) invariant mean $m_{G}$ on $G$.

Examples 2.4. Let the discrete group $Z$ act on the manifold $S^{1}$ by an irrational rotation. Then every orbit of a point $x \in S^{1}$ consists of infinitely many points, and the conditional expectation $E_{\boldsymbol{Z}}$ is well-defined by the integral formula. The positive linear functional $E_{\mathbf{Z}}$ turns out to be the standard integral of continuous functions over $S^{1}$.

Consider the discrete group $\mathbb{Z}$ acting on itself in the natural way. The related conditional expectation $E_{G, a m}$ coincides with the invariant mean $m_{Z}$ selected.

The mapping $E_{G, a m}$ derived from the group action in Example 2.2 by (3) is discontinuous at $(0,0)$ what shows the need of the additional assumption of uniform continuity to the action of $G$ on $X$.

In addition to the proof of well-definedness of this type of conditional expectations, we want to obtain some sufficient conditions for them to be of finite index in the sense of $[3,6]$.

Lemma 2.5. Let $G$ be an (amenable) topological group acting on a locally compact Hausdorff space $X$. Suppose that the orbit $G x$ consists of a finite number of points for every $x \in X$. Then the connected component $H$ of the identity of $G$ acts trivially on $x$, i.e. it belongs to the stabilizer $G_{x}$ of $x \in X$.

Proof. A finite subset of a Hausdorff space $X$ consisting of more than one point is not connected. Consider the mapping $H \rightarrow H / H_{x} \rightarrow H x, h \mapsto h H_{x} \mapsto h x$ where $H_{x}$ is the stabilizer of $x$ under the action of $H$. The first mapping in this combination is an epimorphism, the second one is a homeomorphism. Thus $H x$ is connected and hence coincides with $\{x\}$

Lemma 2.6. Let $G$ be an amenable topological group which acts on a locally compact normal space $X$ in such a way that it generates a conditional expectation $E_{G, a m}$ on $\mathrm{C}_{0}(X)$ (cf. (3)). Suppose for some $x \in X$ there exists an orbit $G x$ consisting of an infinite number of points, and the corresponding mean on $G x$ is normal, namely for every $\varepsilon>0$ there exists a positive function $f$ with $f(x)=1$ and $m_{G x}(f)<\varepsilon$. Then the characteristic number $K\left(E_{G, a m}\right)$ of the conditional expectation $E_{G, a m}$ is infinite.

Proof. The mentioned functions exist by [18: Theorem V.17.4], and they satisfy the inequality $1=f(x) \leq K\left(E_{G, a m}\right) \cdot E_{G, a m}(f)(x)<K\left(E_{G, a m}\right) \varepsilon$. Hence the claim is obvious since $\varepsilon>0$ was arbitrarily chosen

Note that the conditions of Lemma 2.6 are satisfied for Example 2.4.

Theorem 2.7. Let $G$ be an amenable topological group which acts on the locally compact normal space $X$ in such a way that a conditional expectation $E_{G, a m}$ is generated on $\mathrm{C}_{0}(X)$ for a certain (left) invariant mean $m_{G}$ on $G$. Suppose that the corresponding mean on $G x$ is normal there for every $x \in X$. Then there are exactly two cases:

(i) For the connected component of the identity $H$ of $G$ the action of $G$ factorizes into the composition

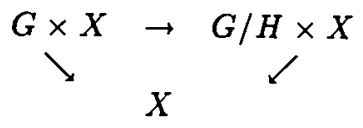


The action of $G$ on $X$ can alternatively be described by the action of the completely disconnected group $G / H$. In this case the characteristic value $K\left(E_{G, a m}\right)$ can in general be finite.

(ii) In all other cases only infinite values $K\left(E_{G ; a m}\right)$ appear.

Proof. First of all, $H$ is a normal subgroup of $G$ by [17: p. 128]. If there is an infinite orbit, then by Lemma 2.6 we obtain the second situation; otherwise we have the first case by Lemma 2.5

The consequence of Theorem 2.7 is that the consideration of conditional expectations of finite index arising from the actions of amenable topological groups on locally compact normal spaces $X$ leads to the investigation of actions of amenable completely disconnected groups on these spaces. We may restrict to the latter kind of actions to look for results. Note that the factor group $G / H$ of a locally compact group by its connected component of the identity $H$ is not necessarily discrete (however, this is true for Lie groups). For example, any infinite product of finite groups is a non-discrete group with trivial connected component. We will, nevertheless, concentrate on the case of finite and discrete group actions during our further considerations. The most promising situation is characterized by the existence of an upper bound for the number of points in any orbit.

Theorem 2.8. Let $X$ be a locally compact Hausdorff space equipped with an action of a finite group $G$. Then for the conditional expectation $E_{G, a m}$ we have the estimate

$$
K\left(E_{G, a m}\right) \leq k:=\max _{x \in X} \#(G x)
$$

If $X$ is a normal space, then we have the equality $K\left(E_{G, a m}\right)=k$.

Proof: Let $x \in X$ be an arbitrary element and $k_{x}=\# G x$. Then

$$
\begin{aligned}
k \cdot E_{G, a m}(f) x & =k \frac{1}{|G|} \sum_{g \in G} f\left(g^{-1} x\right) \\
& =k \frac{1}{|G|} \sum_{[g] \in G / G_{x}}\left|G_{x}\right| \cdot f(g x) \\
& \geq \frac{k}{k_{x}} \sum_{[g] \in G / G_{x}} f\left(g^{-1} x\right) \\
& \geq f(x) .
\end{aligned}
$$

Suppose that $K\left(E_{G, a m}\right)<k$ and $X$ is normal. Take an element $x \in X$ realizing $k_{x}>K\left(E_{G, a m}\right)$. Then there exists a neighbourhood $U_{x}$ of $x$ such that $\left(g U_{x}\right) \cap U_{x}=\emptyset$ for some element $g \in G$ with $g x \neq x$. Since $X$ is supposed to be normal there exists a continuous non-negative function $f$ with support inside $U_{x}$ (cf. [18: Theorem V.17.4). 
Then

$$
\begin{aligned}
K\left(E_{G, a m}\right) \cdot E_{G, a m}(f) x & =K\left(E_{G, a m}\right) \frac{1}{|G|} \sum_{g \in G} f(g x) \\
& =K\left(E_{G, a m}\right) \frac{1}{|G|} \sum_{[g] \in G / G_{x}}\left|G_{x}\right| \cdot f(g x) \\
& <k_{x} \frac{1}{k_{x}} \sum_{[g] \in G / G_{x}} f(g x) \\
& =f(x) .
\end{aligned}
$$

This contradicts the definition of the characteristic number $K\left(E_{G, a m}\right)$, and completes the proof

Lemma 2.9. Suppose a discrete amenable group $G$ acts in a uniformly continuous manner on a locally compact Hausdorff space $X$ in such a way that each orbit has length $\# G x$ with \#Gx $\leq k$ for some fixed $k \in \mathbb{N}$. Then $E_{G, a m}$ is well-defined.

Proof. Let $H \subseteq G$ be a subgroup of finite index and let $T$ be a transversal of $H$, i.e. a choice of representatives in cosets. We have the equality

$$
\begin{aligned}
m_{G}(f(g x)) & =m_{G}\left(\sum_{g_{a} \in T} f\left(h g_{a} x\right) \chi_{g_{a} H}\right) \\
& =\sum_{g_{a} \in T} f\left(g_{a} x\right) \cdot m_{G}\left(\chi_{g_{a} H}\right) \\
& =c \cdot \sum_{g_{a} \in T} f\left(g_{a} x\right) .
\end{aligned}
$$

Set $f=1$. Then for every $x \in X$ the equality $1=m_{G}(1)=c \cdot \#(G / H)$ holds, which implies $c=\#(G / H)^{-1}$ and

$$
m_{G}(f(g x))=\frac{1}{\#(G / H)} \cdot \sum_{g_{a} \in T} f\left(g_{a} x\right) .
$$

Now, fix $\varepsilon>0$ and $x \in X$. Choose $y$ so close to $x$ such that the estimate $|f(g x)-f(g y)|<$ $\varepsilon$ holds for every $g \in G$. Because of the uniform continuity of the action of $G$ on $X$ this choice is possible, since we can take $y$ from such a small neighbourhood $U$ of $x$ that the variation of $f$ is less than $\varepsilon$ on $g_{1} V=V, g_{2} V, \ldots, g_{m} V$ for $\left\{g_{1}=e, g_{2}, \ldots, g_{m}\right\}=T$ and $G_{x} V \subseteq U$. In this situation every $g \in G$ can be decomposed as $g=g_{i} h$ for some $h \in H$, and $g y \in g_{i} V$.

Consequently, the subgroup $D=G_{x} \cap G_{y}$ has finite index and the set of cosets $G / D$ coincides with the set of pairwise intersections $g G_{x} \cap h G_{y}$. Moreover, the index of $D$ in $G$ is not greater than the product of the indices of $G_{x}$ and $G_{y}$ (cf. [12: p. 62]). The functions $\phi(g)=f(g x)$ and $\psi(g)=f(g y)$ are constant on $D$, and so we obtain the equalities

$$
E_{G, a m}(f)(x)=\frac{1}{\#(G / D)} \cdot \sum_{g_{a} \in T} f\left(g_{a} x\right)
$$


and, analogously,

$$
E_{G, a m}(f)(y)=\frac{1}{\#(G / D)} \cdot \sum_{g_{a} \in T} f\left(g_{a} y\right)
$$

for the selected elements $x, y \in X$. Hence

$$
\left|E_{G, a m}(f)(x)-E_{G, a m}(f)(y)\right|<\frac{1}{\#(G / D)} \cdot \#(G / D) \cdot \varepsilon=\varepsilon
$$

and the lemma is proved

Definition 2.10. Suppose an arbitrary topological group $G$ acts in a uniformly continuous manner on a locally compact Hausdorff space $X$ in such a way that each

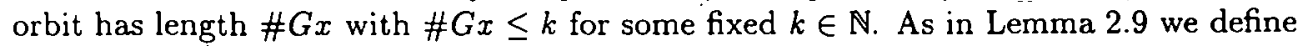
a conditional expectation $E_{G}: \mathrm{C}_{0}(X) \rightarrow E_{G}\left(\mathrm{C}_{0}(X)\right) \subseteq \mathrm{C}_{0}(X)$ by

$$
E_{G}(f)(x)=\frac{1}{\#\left(G / G_{x}\right)} \cdot \sum_{g_{a} \in T_{x}} f\left(g_{a} x\right),(x \in X) .
$$

Precisely the same argument as in Lemma 2.9 applies to prove the following statement.

Lemma 2.11. Suppose a discrete group $G$ acts in a uniformly continuous manner on a locally compact Hausdorff space $X$ in such a way that each orbit has length \#Gx $\leq k$ for some fixed $k \in \mathbb{N}$. Then $E_{G}$ is well-defined.

Theorem 2.12. Suppose a discrete group $G$ acts on a locally compact Hausdorff space $X$ in such a way that $k:=\max \{\#(G x): x \in X\}<+\infty$. If $X$ is a normal space, then $K\left(E_{G}\right)=k$.

Proof. Let $x \in X$ be an arbitrary element and $k_{x}=\# G x$. Then

$$
k \cdot E_{G}(f)(x)=k \frac{1}{k_{x}} \cdot \sum_{g_{a} \in T} f\left(g_{a} x\right) \geq f(x) .
$$

Assume $K\left(E_{G}\right)<k$ for a normal space $X$. Choose a point $x \in X$ with $k_{x}>K\left(E_{G}\right)$. Then we can obtain a neighbourhood $U_{x}$ of $x$ such that $g_{i} U_{x} \cap g_{j} U_{x}=\emptyset$ for $i \neq j$ and $\left\{g_{1}=1, g_{2}, \ldots, g_{m}\right\}=T_{x}$. For a continuous non-negative function $f$ with support inside $U_{x}$ (cf. [18: Theorem V.17.4] for the existence) we have the inequality

$$
K\left(E_{G}\right) \cdot E_{G}(f)(x)=K\left(E_{G}\right) \frac{1}{k_{x}} \cdot \sum_{g_{a} \in T} f\left(g_{a} x\right)<f(x) .
$$

This contradicts the definition of $K\left(E_{G}\right)$, and completes the proof 


\section{Conditional expectations arising from discrete group actions}

At this point we shall investigate conditional expectations of finite index arising from several discrete group actions on compact Hausdorff spaces. The investigations are inspired by the results of Y. Watatani [20: Propositions 2.8.1 and 2.8.2] on actions of finite groups $G$ on compact Hausdorff spaces $X$ giving rise to conditional expectations of finite index $E$ on $\mathrm{C}(X)$,

$$
E_{G}(f)(x)=\frac{1}{|G|} \sum_{g \in G} f(g x) \quad(x \in X),
$$

which are sometimes non-algebraically of finite index. We would like to give a more detailed analysis of actions of amenable discrete groups $G$ on compact Hausdorff spaces $X$ for which the orbit of every $x \in X$ is finite. For the definition of a corresponding conditional expectation $E_{G}$ on the $\mathrm{C}^{*}$-algebra $\mathrm{C}(X)$ we refer to Lemma 2.10 and 2.12. The goal is to give a more detailed analysis of the phenomena appearing from the view point of Hilbert $\mathrm{C}^{*}$-module theory adapting techniques from [20].

First we want to give two examples of conditional expectations of finite index which are not algebraically of finite index in the sense of $Y$. Watatani's definition. They are derived from [20: Propositions 2.8.1 and 2.8.2] as special cases giving new arguments for proving their non-triviality.

Example 3.1. Let $X$ be the compact Hausdorff subspace $X=\left\{t \cdot \mathrm{e}^{2 k \pi o v e r n i}: k=\right.$ $1, \ldots, n, t \in[0,1]\}$ of $\mathbb{C}$ which can be considered as a starlike arrangement of $n$ copies of the unit interval with zero as the common element. Let $A$ be the $\mathrm{C}^{*}$-algebra $\mathrm{C}(X)$ of all continuous functions on $X$. Consider the normal conditional expectation $E$ on $A$ defined by taking the mean with respect to the cyclic group action

$$
E(f)\left(x_{1}\right)=\ldots=E(f)\left(x_{n}\right)=\frac{f\left(x_{1}\right)+\ldots+f\left(x_{n}\right)}{n} \quad\left(x_{i} \in[0,1]_{i}, i=1, \ldots, n\right) .
$$

The image $C^{*}$-algebra $B$ can be identified with the subset of functions of $A$

$$
\left\{f \in A: f\left(x_{1}\right)=\ldots=f\left(x_{n}\right) \text { for } x_{i} \in[0,1]_{i}(i=1, \ldots, n)\right\}
$$

inheriting the $\mathrm{C}^{*}$-structure from $A$. Note that $\left\{A, E\left(\langle., .\rangle_{A}\right)\right\}$ is not a self-dual Hilbert $B$-module since the bounded $B$-linear mapping

$$
E\left(f_{0}^{*} f\right)\left(x_{1}\right)=\ldots=E\left(f_{0}^{*} f\right)\left(x_{n}\right)=\frac{f_{0}^{*}\left(x_{1}\right) f\left(x_{1}\right)+\ldots+f_{0}^{*}\left(x_{n}\right) f\left(x_{n}\right)}{n}
$$

defined for $f \in A$ with $x_{i} \in[0,1]_{i} \quad(i=1, \ldots, n)$ and

$$
f_{0}(x)= \begin{cases}1 & \text { if } x_{1} \in(0,1)_{1} \\ -1 & \text { if } x_{2} \in(0,1)_{2} \\ 0 & \text { elsewhere }\end{cases}
$$


maps $A$ into $B$, but $f_{0}$ does not belong to $A$. Consequently, the Hilbert $B$-module $\left\{A, E\left(\langle., .\rangle_{A}\right)\right\}$ cannot be finitely generated and projective, and $E$ is not of finite index in the sense of Y. Watatani's algebraic definition. Nevertheless, $E$ has a very good property: the mapping $\left(n \cdot E-\mathrm{id}_{M}\right)$ is obviously completely positive. Besides this, a careful analysis performed below yields the $B$-reflexivity of the Hilbert $B$-module $\left\{A, E\left(\langle\cdot, \cdot\rangle_{A}\right)\right\}$. The characteristic number of $E$ is $K(E)=n$.

Now let us consider the index value. For this we extend $E$ to its canonical normal conditional expectation $E^{* *}: A^{* *} \rightarrow B^{* *} \subseteq A^{* *}$ (see $[4,18]$ ) and count the index of the extended conditional expectation $E^{* *}$ as an element of the center of $A^{* *}$. The corresponding constants

$$
K\left(E^{* *}\right)=K(E)=\inf \left\{K:\left(K \cdot E-\mathrm{id}_{A}\right) \text { is positive on } A\right\}
$$

and

$$
L\left(E^{* *}\right)=L(E)=\inf \left\{L:\left(L \cdot E-\mathrm{id}_{A}\right) \text { is completely positive on } A\right\}
$$

are preserved by monotonicity [14].

The discrete part of the bidual linear space and $\mathrm{W}^{*}$-algebra $A^{* *}$ of $A$ is isomorphic to $l_{\infty}(X)$ by the Gel'fand theorem. The formula defining $E^{* *}$ is the same as for $E$. The index can be algebraically counted. The resulting value is

$$
\operatorname{Ind}(E)=\operatorname{Ind}\left(E^{* *}\right)_{d i s c r}=\left\{\begin{array}{ll}
2 & \text { if } x_{i} \neq 0 \quad(i=1, \ldots, n) \\
1 & \text { if } x=0
\end{array} \quad \in l_{\infty}(X),\right.
$$

and $\operatorname{Ind}(E) \notin A \hookrightarrow A_{d i s c r}^{* *}$. Note that the index value $\operatorname{Ind}(E) \in A_{d i s c r}^{* *}$ gives rise to a positive mapping $\left(\operatorname{Ind}(E) \cdot E^{* *}-\mathrm{id}_{A_{\text {di.cr }}^{* . *}}\right)$ on $A_{\text {discr }}^{* *}$.

Since $A^{* *}$ is commutative (hence, type I) let us look at the non-discrete part of it. By the decomposition theory of commutative $W^{*}$-algebras it is a direct sum of $W^{*}$ algebras $L^{\infty}([0,1], \lambda)$, where $\lambda$ denotes the Lebesgue measure, (cf. [18]). Consider the canonical embedding $A=\mathrm{C}(X) \subset L^{\infty}(X, \lambda) \cong \sum \oplus L^{\infty}\left([0,1]_{i}, \lambda\right)$. Again, the index of $E^{* *}$ reduced to $L^{\infty}(X, \lambda)$ can be found; it equals the function $f(x) \equiv 2$, which is different from the value obtained for the discrete part of $\operatorname{Ind}\left(E^{* *}\right)$.

Example 3.2. Consider two copies of the usually topologized interval $[-1,1]$ and denote their disjoint union by $X$. Let the cyclic group $G=\mathbb{Z}_{4}$ act on $X$ in such a way that the elements of $X=[-1,1]_{1} \cup[-1,1]_{2}$ are mapped by the rule

$$
x \in[-1,1]_{1} \rightarrow-x \in[-1,1]_{2} \quad \text { and } \quad x \in[-1,1]_{2} \rightarrow x \in[-1,1]_{1} \text {. }
$$

The orbits of all points $x \in X \backslash\left\{0 \in[-1,1]_{1}, 0 \in[-1,1]_{2}\right\}$ have length 4 , the two exceptional points have an orbit of length 2 . Now, switch back to the derived action of $G$ on the corresponding unital $C^{*}$-algebra $A=\mathrm{C}(X)$ and consider the canonically defined conditional expectation

$$
E(f)(x)=\frac{1}{4}\left(f(e x)+f(g x)+f\left(g^{2} x\right)+f\left(g^{3} x\right)\right)
$$


(where $g \in \mathbb{Z}_{4}$ is a generator) which possesses the characteristic constant $K(E)=4$. If we consider a bounded $B$-linear functional on the Hilbert $B$-module $\{A, E(\langle\cdot, \cdot\rangle)\}$ defined by the rule $E\left(f_{1}^{*} f\right)$ for $f \in A$ and

$$
f_{1}(x)= \begin{cases}1 & \text { if } x \in[-1,0)_{1} \\ -1 & \text { if } x \in(0,1]_{1} \\ 0 & \text { if elsewhere }\end{cases}
$$

we obtain the non-self-duality of $\{A, E(\langle\cdot, \cdot\rangle)\}$ as Hilbert $B$-module again. Therefore, $E$ cannot be algebraically of finite index, despite the non-existence of fixed points under the action of $G$ on $X$.

Two other examples show that actions of infinite amenable discrete groups can yield non-trivial conditional expectations of finite index, as well as mappings $E_{G}$ with images outside the $\mathrm{C}^{*}$-algebra $\mathrm{C}(X)$. In other words, we must always keep in mind the elements of the construction. Very often we can adapt examples with locally compact spaces $X$ to the compact situation switching to its one-point-compactification $\alpha X$. However, additional fixed points of the action of $G$ can appear during this process.

Example 3.3. Let $G=\oplus_{i=1}^{\infty}\left(\mathbb{Z}_{2}\right)_{i}$, an infinite discrete group. Let $g_{i}$ be the generator of the $i$-th copy of $\mathbb{Z}_{2}$. Let $Y:=\left\{\frac{1}{n}: n \in \mathbb{N}\right\} \cup\{0\}$ and $X:=Y \times I$, where $I=[0,1]$. If the canonical pointwise identification maps $a_{j}^{i}$ of $\left\{\frac{1}{i}\right\} \times I$ and $\left\{\frac{1}{j}\right\} \times I$ are used, we can define the action of $G$ on $X$ by

$$
g_{i}(x)= \begin{cases}a_{2 i}^{2 i-1}(x) & \text { if } x \in\left\{\frac{1}{2 i-1}\right\} \times I \\ a_{2 i-1}^{2 i}(x) & \text { if } x \in\left\{\frac{1}{2 i}\right\} \times I \\ x & \text { elsewhere. }\end{cases}
$$

Then the cardinality of any orbit is less or equal to 2 ; however, the action of $G$ does not factorize through any action of any compact or finite group. Now, define a natural conditional expectation $E$ on $A=\mathrm{C}_{0}(X)$ by the formula

$$
\begin{aligned}
E(f)(x) & =\lim _{n \rightarrow \infty} \frac{1}{2^{n}} \sum_{g \in \oplus_{i=1}^{n}\left(\mathbf{Z}_{2}\right)_{i}} f(g x) \\
& =\lim _{n \rightarrow \infty} \frac{1}{2^{n}} \cdot 2^{(n-1)}\left(\sum_{g \in \mathbf{Z}_{2}} f(g x)\right) \quad(x \in X) \\
& =\frac{f(e \circ x)+f(g \circ x)}{2} .
\end{aligned}
$$

We obtain a natural conditional expectation of finite index with structural constant $K(E)=2$, which is algebraically of finite index, too. The intrinsic point is that $E$ can be alternatively induced by an action of the group $\mathbb{Z}_{2}$ on $X$, which is different from the group action we started with, despite the fact that the original group action cannot be replaced by any finite group action. 
Example 3.4. Let $G=\mathbb{Z}$ act on $X=\mathbb{R}$ as shifts. Let $f: \mathbb{R} \rightarrow \mathbb{C}$ be the following positive continuous even function:

$$
f(x)= \begin{cases}1 & \text { if } x \in\left[n+\frac{1}{n+1}, n+1-\frac{1}{n+1}\right] \quad(n \geq 0) \\ 0 & \text { if } x=n \quad(n \geq 0) \\ \text { linear } & \text { if } x \text { in remaining intervals. }\end{cases}
$$

Then $E(f)(0+i)=0$ for any $i \in \mathbb{Z}$. For $x \notin \mathbb{Z}$ there exists a number $j_{0} \in \mathbb{N}$ such that $f(x+j)=1$ for $j$ with $|j|>j_{0}$. Hence

$$
\begin{aligned}
E(f)(x) & =\lim _{n \rightarrow \infty} \frac{1}{2 n+1} \sum_{j=-n}^{j=n} f(x+i) \\
& =\lim _{n \rightarrow \infty} \frac{1}{2 n+1} \sum_{j=-k_{0}}^{k_{0}} f(x+i)+\lim _{n \rightarrow \infty} \frac{1}{2 n+1} \sum_{k_{0}<|j| \leq n} f(x+i) \\
& =0+\lim _{n \rightarrow \infty} \frac{2\left(n-k_{0}\right)}{2 n+1}=1
\end{aligned}
$$

Summing up we obtain

$$
E(f)(x)=\left\{\begin{array}{ll}
1 & \text { if } x \notin \mathbb{Z} \\
0 & \text { if } x \in \mathbb{Z}
\end{array} .\right.
$$

which is a discontinuous function.

The following example describes an action of an amenable group $G$ on a (locally) compact Hausdorff space $X$, which induces a non-trivial (i.e. non-state) conditional expectation $E$ on the $C^{*}$-algebra $\mathrm{C}_{0}(X)$ which cannot be induced by an action of a finite group on $X$.

Example 3.5. Let $G=\oplus_{k=1}^{\infty} \mathbb{Z}_{k}$, a separable discrete group. Denote by $g_{k}$ the generator of the $k$-th summand $\mathbb{Z}_{k} \subset G$. Let $X$ be the infinite disjoint union (summing over $k \in \mathbb{N}$ ) of disjoint unions of $k$ copies of the interval $I_{k}=[0,1]$ respectively. If $a_{j}^{i}(k)$ denotes the pointwise identification function of the intervals $I_{k}^{i}$ and $I_{k}^{j}$ at the $k$ th position, then we describe the action of $G$ on $X$ by the formula

$$
g_{k}(x)= \begin{cases}a_{i+1 \bmod k}^{i}(k)(x) & \text { if } x \in I_{k}^{i} \\ x & \text { elsewhere. }\end{cases}
$$

Of course, in this situation the characteristic number $K(E)=+\infty$.

Theorem 3.6. Let $X$ be a compact Hausdorff space and $G$ a discrete group acting in a uniformly continuous manner on $X$ so that all orbits consist of the same finite .number of points. Then the Hilbert $\mathrm{C}^{G}(X)$-module $\{C(X), E(\langle\cdot, \cdot\rangle)\}$ is finitely generated and projective.

Proof. We want to imitate the original proof of $\mathrm{Y}$. Watatani-in the case of a free action of a finite group [20: Proposition 2.8.1]. By [18: Theorem VI.22.8] every compact Hausdorff space is normal. Thus Urysohn's lemma can be used (cf. [18: Prop. V.17.4]). However, first we need the following two lemmata. 
Lemma 3.7. Let $X$ be a compact Hausdorff space and $G$ be a discrete group acting in a uniformly continuous manner on $X$ so that all orbits consist of the same finite number of points. Then for $x \in X$ and $g \in G_{x}$ there exists an open neighbourhood $U_{x}$ of $x$ on which $g$ acts as the identity map.

Proof. Consider the stabilizer subgroup $G_{x} \subset G$. It is of finite index, and the index is $G: G_{x}=\#(G x)=n$. Let $x_{1}, \ldots, x_{n} \in X$ be the orbit of $x$ and $h_{i} \in G$ be elements such that $h_{i} x=x_{i}$. Fix $g_{0} \in G_{x}$ and assume that every neighbourhood $U_{x}$ of $x$ contains some point $y \in U_{x}$ such that $g_{0} y \neq y$. Fix some neighbourhoods $U_{x_{i}}$ of the points $x_{i}$ satisfying $U_{x_{i}} \cap U_{x_{j}}=\emptyset$ for $i \neq j$. There exists a neighbourhood $V_{x} \subseteq U_{x}$ of $x$ such that $h_{i}\left(V_{x}\right) \subseteq U_{x_{i}}$. By assumption $G$ acts on $X$ uniformly continuously, so let $W_{x} \subseteq V_{x}$ be a neighbourhood of $x$ such that $g\left(W_{x}\right) \subseteq V_{x}$ for every $g \in G_{x}$. For $y \in W_{x}$ with $g_{0} y \neq y$ the orbit $G y$ contains at least $n+1$ distinct points: $\left\{g_{i} y: i=1, \ldots, n\right\} \in U_{x_{i}}$ and $\left\{y, g_{0} y\right\} \in V_{x}$. This contradicts with the boundedness of orbits by $n$, and proves the lemma

Lemma 3.8. Let $X$ be a compact Hausdorff space and $G$ be a discrete group acting in a uniformly continuous manner on $X$ so that all orbits consist of equal finite numbers of points. For any $x \in X$ there exists a neighbourhood $V_{x}$ such that the action of $G_{x}$ on $V_{x}$ is the identical map.

Proof. For cvery $g \in G_{x}$ set $U_{x}(g)=\{y \in X: g y=y\}$. Suppose at the contrary that the set $\cap_{g \in G_{x}} U_{x}(g)$ does not contain any neighbourhood of the point $x$, i.e. every neighbourhood $U_{x}$ of $x$ contains some point $z$ such that for some $g_{z} \in G_{x}$ we have $g_{z} z \neq z$. Consider a neighbourhood $V_{x}$ of $x$ and neighbourhoods $\left\{U_{g_{i} x}\right\}$ for fixed representatives $g_{i} \in G\left(g_{i} \neq e\right)$ of the cosets of $G / G_{x}$ such that their pairwise intersection is empty and $g_{i}\left(V_{x}\right) \subseteq U_{h_{i} x}$ (cf. the proof of Lemma 3.7). By assumption $G$ acts in a uniformly continuous manner on $X$, i.e. there exists a neighbourhood $W_{x} \subseteq V_{x}$ of $x$ such that $g\left(W_{x}\right) \subseteq V_{x}$ for every $g \in G_{x}$. Define $U_{x}=\cup_{g \in G_{x}} g\left(W_{x}\right) \subseteq V_{x}$. It is an $G_{x}$-invariant open neighbourhood of $x \in X$. By construction the assumption $\dot{g}_{z} z \neq z$ for some $z \in U_{x}, g_{z} \in G_{x}$ leads to an orbit of $z$ consisting of at least $n+1$ points, which contradicts the supposition

Let $U_{x} \subset V_{x}$ be neighbourhoods of $x$ such that the action of $G_{x}$ on $V_{x}$ is the identical map. Then there exists a function $f_{x} \in \mathrm{C}(X)$ such that supp $f_{x} \subset V_{x}$ and $f_{x} \mid U_{x}=1$. For any $g \in G$ we have either $\left(g V_{x}\right) \cap V_{x}=\emptyset$ or $g V_{x} \equiv V_{x}$, which implies that

$$
\alpha_{g}\left(f_{x}\right) \cdot f_{x}= \begin{cases}\alpha_{g}\left(f_{x}\right)^{2} & \text { if } g x=x \\ 0 & \text { otherwise }\end{cases}
$$

where $\alpha$ denotes the action of the group $G$ on functions, $\alpha_{g}(f)(x)=f(g x)$. Fix a finite covering $\left\{U_{x_{1}}, \ldots, U_{x_{k}}\right\}$ of $X$ by such type of sets and set

$$
v=\sum_{i=1}^{k} f_{x_{i}} \geq 1, \quad u_{i}=v^{-\frac{1}{2}}\left(f_{x_{i}}\right)^{\frac{1}{2}} \in \mathrm{C}(X) .
$$

Note that for representatives $g_{i}$ of the cosets of $G / G_{x}$ we have a well-defined mapping

$$
E_{G}(f)(x)=\frac{1}{\#(G x)} \sum_{i=1}^{n} f\left(g_{i} x\right)
$$


for every $x \in X, f \in \mathrm{C}(X)$ due to the fixed length of orbits on $X$ and Lemma 3.7. It is a conditional expectation on $\mathrm{C}(X)$. Furthermore, for every $f \in \mathrm{C}(X)$ we obtain the equalities

$$
\sum_{i=1}^{n} u_{i} \cdot E_{G}\left(u_{i}^{*} f\right)=\sum_{i=1}^{n}\left(\frac{1}{n} \sum_{j=1}^{n} u_{i}(x) u_{i}^{*}\left(g_{j} x\right) f\left(g_{j} x\right)\right)=f, \quad E_{G}\left(u_{i}^{*} u_{j}\right)=\delta_{i, j}
$$

Hence, $\left\{u_{1}, \ldots, u_{n}\right\}$ is a quasi-basis of the Hilbert $\mathrm{C}^{G}(X)$-module $\left\{\mathrm{C}(X), E_{G}(\langle.,\rangle).\right\}$, which is therefore shown to be finitely generated and projective

Let us comment that finite generatedness and projectivity implies self-duality for the Hilbert $C^{G}(X)$-module $\left\{\mathrm{C}(X), E_{G}(\langle.,\rangle).\right\}$. In the case of general group actions we cannot expect the resulting action on the $\mathrm{C}^{*}$-algebra $A=\mathrm{C}(X)$ to lead to a conditional expectation $E_{G}$ on $A$ turning it into a self-dual Hilbert $C^{G}(X)$-module. However, if $K\left(E_{G}\right)<\infty$ exists (i.e. when $A$ is complete with respect to the norm given by the corresponding $C^{*}$-valued inner product) we can expect that it might be $C^{*}$-reflexive, i.e. the $\mathrm{C}^{*}$-bidual Hilbert $\mathrm{C}^{G}(X)$-module $A^{\prime \prime}$ of $A$ coincides with $A$, where $A^{\prime}$ denotes the dual Banach $C^{G}(X)$-module of bounded $C^{G}(X)$-homomorphisms from $A$ to $C^{G}(X)$. We can show the following fact:

Theorem 3.9. Let $X$ be a compact Hausdorff space and $G$ a discrete group acting in a uniformly continuous manner on $X$. If $G$ acts in such a way that the lengths of all orbits are uniformly bounded by some number $n$ and the number of points whose orbit is shorter than $n$ is finite, then the Hilbert $\mathrm{C}^{G}(X)$-module $\left\{\mathrm{C}(X), E_{G}(\langle.,\rangle).\right\}$ is $\mathrm{C}^{G}(X)$-reflexive.

Proof. Suppose that $X$ has isolated points $\left\{x_{1}, \ldots, x_{k}\right\}$ with orbit shorter than $n$. By assumption this set is invariant under the action of $G$ on $X$ as well as its complement $X^{\prime} \subset X$. The Hilbert $\mathrm{C}^{G}(X)$-module $\left\{\mathrm{C}(X), E_{G}(\langle\cdot, \cdot\rangle)\right\}$ splits into the direct sum $\mathrm{C}\left(X^{\prime}\right) \oplus \mathrm{C}\left(\left\{x_{1}, \ldots, x_{k}\right\}\right)$, and the theorem is obvious for the second direct summand. So we may assume that $X$ does not contain any isolated point with orbit shorter than $n$ switching from the $\mathrm{C}^{*}$-algebra of coefficients $\mathrm{C}^{G}(X)$ to the smaller $\mathrm{C}^{*}$ algebra of coefficients $C^{G}\left(X^{\prime}\right)$.

We start with a description of the $\mathrm{C}^{*}$-dual Banach $\mathrm{C}^{G}(X)$-module $A^{\prime}$. Let $x_{1}, \ldots, x_{n}$ be the points whose orbits have a length less than $n$. We can take some open neighbourhoods $U_{1}, \ldots, U_{n}$ of these points such that, firstly, these neighbourhoods are invariant under the action of elements of $G_{x_{i}}$ (i.e. under the action of those which do not move the point $\left.x_{i}, i=1, \ldots, n\right)$ and, secondly, such that if for some $h \in G$ we have $h x_{i}=x_{j}$, then $h U_{i}=U_{j}$. Denote the compact space $X \backslash\left(U_{1} \cup \ldots \cup U_{n}\right)$ by $Y$. Note that $Y$ is also $G$-invariant. Let $F \in A^{\prime}$ be an $C^{G}(X)$-valued functional on $\mathrm{C}(X)$. Due to commutativity we can consider its restriction to the Hilbert $\mathrm{C}^{G}(Y)$-module $\left\{\mathrm{C}(Y), E_{G}(\langle.,\rangle).\right\}$. More precisely, for $g \in \mathrm{C}(Y)$ we can take its extension $\tilde{g} \in \mathrm{C}(X)$ and define $\left.F\right|_{Y}$ by $\left.F\right|_{Y}(g)=\left.F(\tilde{g})\right|_{Y}$. The restriction defined in this way does not depend on the choice of extension. If $Y^{\prime} \supset Y$ is also a compact $G$-invariant subset of $X$ not containing the points $x_{1}, \ldots, x_{n}$, then obviously $\left.\left(\left.F\right|_{Y^{\prime}}\right)\right|_{Y}=\left.F\right|_{Y}$. However, since every point in $Y$ has an orbit of the same length $n$ we can use the self-duality of the 
Hilbert $\mathrm{C}^{G}(Y)$-module $\left\{\mathrm{C}(Y), E_{G}(\langle.,\rangle).\right\}$ to conclude that $\left.F\right|_{Y} \in \mathrm{C}(\mathrm{Y})$ by Theorem 3.6. Denote by $\mathrm{C}\left(X \backslash\left\{x_{1}, \ldots, x_{n}\right\}\right)$ the set of continuous functions on the non-compact space $X \backslash\left\{x_{1}, \ldots, x_{n}\right\}$. Then taking restrictions we get the map

$$
A^{\prime} \longrightarrow \mathrm{C}\left(X \backslash\left\{x_{1}, \ldots, x_{n}\right\}\right) \text {. }
$$

If $F_{1}, F_{2} \in A^{\prime}$ coincide on $X \backslash\left(x_{1}, \ldots, x_{n}\right)$, then (as they are mappings of continuous functions to continuous functions) they coincide on $X$, hence the map (4) is a monomorphism.

Now we study local properties of functionals from $A^{\prime}$ near the singular points $x_{1}, \ldots, x_{n}$. Let $x_{0}$ be one of these points. There exists a neighbourhood $U_{x_{0}}$ of the point $x_{0}$ such that for $g x_{0}=x_{0}$ the set identity $g U_{x_{0}}=U_{x_{0}}$ follows. The group $G_{x_{0}}$ contains a normal subgroup $G_{0}$ of elements which leave the points of $U_{x_{0}}$ invariant. Fix one element $g_{i}$ in every coset $G / G_{x_{0}}$. Then outside the point $x_{0}$ the action of a functional $F \in A^{\prime}$ is given by the equality

$$
F(f)(x)=\frac{1}{n} \sum_{i=1}^{n} F^{*}\left(g_{i} x\right) \cdot f\left(g_{i} x\right),
$$

and this action is extended to the point $x_{0}$ by continuity. Let $x_{0}=x^{0}, x^{1}, \ldots, x^{k-1}$ be the orbit of $x_{0}$. Then the sum (5) can be written as

$$
F(f)(x)=\frac{1}{n} \sum_{j=0}^{k-1}\left(\sum_{i: g_{i} x_{0}=x^{j}} F^{*}\left(g_{i} x\right) \cdot f\left(g_{i} x\right)\right)
$$

Taking the norm limit which exists by assumption we obtain

$$
F(f)\left(x_{0}\right)=\lim _{x \rightarrow x_{0}} \frac{1}{n} \sum_{j=0}^{k-1}\left(\sum_{i: g_{i} x_{0}=x^{j}} F^{*}\left(g_{i} x\right) \cdot f\left(x^{j}\right)\right),
$$

which implies the existence of the limit

$$
\frac{1}{n} \lim _{x \rightarrow x_{0}} \sum_{i: g_{i} x_{0}=x^{j}} F\left(g_{i} x\right)
$$

for the special function $f \equiv 1$ of $\mathrm{C}(X)$ and for every $x \in X \backslash\left\{x_{1}, \ldots, x_{n}\right\}$. Recall that the function $F$ was only defined outside the point $x_{0}$. If we want to write the action of $F$ on the Hilbert $\mathrm{C}^{G}(Y)$-module $\left\{\mathrm{C}(Y), E_{G}(\langle\cdot, \cdot\rangle)\right\}$ in the form of $(5)$ everywhere on $X$ we have to define the function $F$ at the point $x_{0}$ by

$$
F\left(x_{0}\right)=\frac{1}{n} \lim _{x \rightarrow x_{0}} \sum_{i: g_{i} x_{0}=x^{j}} F\left(g_{i} x\right) .
$$

Lemma 3.10. The module $A^{\prime}$ is isomorphic to the module of all bounded functions $F$ on $X$ which are continuous on $X \backslash\left\{x_{1}, \ldots, x_{n}\right\}$ and which satisfy the limit condition (6). 
Proof. The remaining aspect is to prove that the image of the monomorphism (4) consists of bounded functions. Suppose the contrary. Then we can find some point $\bar{x}$ such that $|F(\bar{x})|>n \cdot\|F\|$, where $\|F\|$ defines the norm of $F$ in the $C^{*}$-dual Banach $C^{G}(X)$-module $A^{\prime}$. Moreover, we can choose a neighbourhood $U_{\bar{x}}$ of $\bar{x}$ such that the intersection $U_{\bar{x}} \cap g_{i} U_{\bar{x}}$ is empty whenever $g_{i} \bar{x} \neq \bar{x}$. Consider a function $f \in \mathrm{C}(X)$ such that $f(\bar{x})=1$ and supp $f \subset U_{\bar{x}}$. For $f$ we derive $F(f)(\bar{x})=\frac{1}{n} F^{*}(\bar{x}) \cdot f(\bar{x})$, from equality (5), and the resulting inequality

$$
|F(f)(\bar{x})|=\frac{1}{n}\left|F^{*}(\bar{x})\right| \cdot|f(\bar{x})|=\frac{1}{n}|F(\bar{x})|>\|F\|,
$$

gives rise to a contradiction

Now, equipped with a description of the elements in the $\mathrm{C}^{*}$-dual module $A^{\prime}$ of the Hilbert $\mathrm{C}^{G}(Y)$-module $\left\{\mathrm{C}(Y), E_{G}(\langle.,\rangle).\right\}$ we can finally describe the corresponding $\mathrm{C}^{*}$ bidual module $A^{\prime \prime}$. Since $A^{\prime \prime}$ is a canonical subset of $A^{\prime}$ and since the $\mathrm{C}^{*}$-valued inner product of every Hilbert $C^{*}$-module can be continued to a $C^{*}$-valued inner product on its $\mathrm{C}^{*}$-bidual Banach $\mathrm{C}^{*}$-module turning the latter into a Hilbert $\mathrm{C}^{*}$-module [13], we only have to find out the kind of functions of $A^{\prime}$ to which the $\mathrm{C}^{G}(X)$-valued inner product can be extended to. Consider a function $F$ from $A^{\prime} \cap A^{\prime \prime}$. Without loss of generality (adding some continuous function from $A$ ) we can assume that $F\left(x_{0}\right)=0$. Then the value of the $C^{G}(X)$-valued inner product of $F$ with itself has to be contained in $\mathrm{C}^{G}(X)$ and is given by the formula

$$
\langle F, F\rangle(x)=E\left(|F(x)|^{2}\right)=\frac{1}{n} \sum_{i: g_{i} x_{0}=x^{j}}\left|F\left(g_{i} x\right)\right|^{2}
$$

for every $x \in X$. But, since $\langle F, F\rangle\left(x_{0}\right)=0$ the assumption $F \in A^{\prime \prime}$ on $F$.yields that $\|\cdot\|-\lim _{x \rightarrow x_{0}} F\left(g_{i} x\right)=0$ for every summand in equality (7). Consequently, by equality (6) we obtain the continuity of $F$ at $x_{0}$, and $F$ has to belong to $A$

Remark. Let $X$ be a compact Hausdorff space. Suppose a discrete group $G$ acts on $X$ in such a way that the length of any orbit does not exceed $n$. Denote by $Z \subset X$ the set of points whose orbits have a length shorter than maximal. If the set $X \backslash Z$ is dense in $X$, then in a similar way we can prove that the dual Banach $\mathrm{C}^{G}(X)$-module $A^{\prime}$ is isomorphic to some $C^{*}$-subalgebra of the algebra $\mathrm{C}_{b}(X \backslash Z)$ of all bounded continuous functions on $X \backslash Z$. The $\mathrm{C}^{*}$-algebra $\mathrm{C}_{0}(X \backslash Z)$ of continuous functions on $X$ vanishing on $Z$ is an ideal in $\mathrm{C}_{b}(X \backslash Z)$. It would be interesting to describe the structure of the factor-algebra $A^{\prime} / \mathrm{C}_{0}(X \backslash Z)$ in terms of the group action.

Acknowledgement. Our research was supported by the Volkswagen-Stiftung (Research-in-Pairs program at Oberwolfach). The authors are very grateful to the staff of the Mathematisches Forschungsinstitut Oberwolfach for the excellent conditions which supported the fruitful work.

The first author is grateful to Eberhard Kirchberg for his collaboration and fruitful discussions leading to the progress made in [6]. The authors are indebted to G. K. Pedersen, M. A. Rieffel and Y. Watatani for useful remarks and to the referees for their valuable comments on the first version of the present paper. We wish to thank $\mathrm{C}$. Abbey for the final proof-reading of the manuscript. 
Preliminary joint research was partially supported by the local DAAD project "Noncommutative geometry" at the University of Leipzig in conjunction with the Moscow State University. Research by the second and third authors was partially assisted by the RBRF (Grant 96-01-00182-a). The research of the third author was partially supported by the INTAS (Project 94-3420 "Geometry of discrete groups").

\section{References}

11 Andruchow, E. and D. Stojanoff: Geometry of conditional expectations of finite index. Internat. J. Math. 5 (1994), $169-178$.

[2] Andruchow, E. D. and Stojanoff: Conditional expectations of finite weak index. Preprint 1995 and private communication.

[3] Baillet, M., Denizeau, Y. and J.-F. Havet: Indice d'une esperance conditionelle. Compos. Math. 66 (1988), $199-236$.

[4] Bing-Ren, Li: Introduction to Operator Algebras. Singapore: World Scientific 1992.

[5] Choda, H.: A correspondence between subgroups and subalgebras in a $C^{*}$-crossed product. Part I. Proc. Sympos. Pure Math. 38 (1982), 375 - 377.

[6] Frank, M. and E. Kirchberg: On conditional expectations of finite index. J. Oper. Theory (accepted Sept. 1997).

[7] Greenleaf, F. G.: Invariant Means on Topological Groups and Their Applications. New York: Van Nostrand 1969.

[8] Jolissaint, P.: Indice d'esperances conditionnelles et algèbres de von Neumann finies. Math. Scand. 68 (1991), $221-246$.

[9] Jones, V.: Index of subfactors. Invent. Math. 41 (1981), $1-25$.

[10] Khoshkam, Mahmood: Hilbert $C^{*}$-modules and conditional expectations on crossed products. J. Austral. Math. Soc. (Series A) 61 (1996), 106 - 118.

[11] Kurosh, A. G.: The Theory of Groups. New York: Chelsea 1960.

[12] Paschke, W. L.: Inner product modules over $B^{*}$-algebras. Trans. Amer. Math. Soc. 182 (1973), $443-.468$.

[13] Paschke, W. L.: The double B-dual of an inner product module over a $C^{*}$-algebra $B$. Canad. J. Math. 26 (1974), $1272-1280$.

[14] Pedersen, G. K.: $C^{*}$-algebras and Their Automorphism Groups. London: Academic Press 1979.

[15] Pimsner, M. and S. Popa: Entropy and index for subfactors. Ann. Scient. Ec. Norm. Sup. 19 (1986), 57 - 106.

[16] Pontryagin, L. S.: Topological Groups. New York: Gordon and Breach 1966.

[17] Rieffel, M. A.: Induced representations of $C^{*}$-algebras. Adv. Math. 13 (1974), 176 - 257.

[18] Rinow, W.: Lehrbuch der Topologie. Berlin: Dt. Verlag Wiss. 1975.

[19] Takesaki, M.: Theory of Operator Algebras. Vol. I. New York: Springer-Verlag 1979.

[20] Watatani, Y.: Index for $C^{*}$-subalgebras. Memoirs Amer. Math. Soc. $4 \check{2} 4$ (1990), 83 pp.

Received 09.01.1997; in revised form 30.09.1997. 